\title{
New evolutionary models for massive ZZ Ceti stars
}

\section{The effects of crystallization on their pulsational properties}

\author{
A. H. Córsico ${ }^{1,2, \star}$, L. G. Althaus ${ }^{1,2,3, \star}$, M. H. Montgomery ${ }^{4}$, E. García-Berro ${ }^{3,5}$, and J. Isern ${ }^{3,6}$ \\ 1 Facultad de Ciencias Astronómicas y Geofísicas, Universidad Nacional de La Plata, Paseo del Bosque s/n, \\ (1900) La Plata, Argentina \\ e-mail: acorsico@fcaglp.unlp.edu.ar \\ 2 Instituto de Astrofísica La Plata, IALP, CONICET, Argentina \\ 3 Departament de Física Aplicada, Universitat Politècnica de Catalunya, Escola Politècnica Superior de Castelldefels, \\ Av. del Canal Olímpic, s/n, 08860 Castelldefels, Spain \\ e-mail: [leandro;garcia]@fa.upc.es \\ ${ }^{4}$ Department of Astronomy, University of Texas, Austin, TX 78712, USA \\ e-mail: mikemon@ast.cam.ac.uk \\ 5 Institut d'Estudis Espacials de Catalunya, Ed. Nexus, c/Gran Capità 2, 08034 Barcelona, Spain \\ 6 Institut de Ciències de l'Espai (CSIC), Spain \\ e-mail: isern@ieec.fcr.es
}

Received 15 April 2004 / Accepted 12 August 2004

\begin{abstract}
In view of recent claims that asteroseismology could supply invaluable insight into the crystallization process occurring in the interiors of massive white dwarf stars, we present in this work new pulsational calculations for improved carbonoxygen DA white dwarf models suitable for the study of massive ZZ Ceti stars. The background models employed in this study, presented in detail in a recent paper by Althaus et al. (2003, A\&A, 404, 593), are the result of the complete evolution of massive white dwarf progenitors from the zero-age main sequence through the Asymptotic Giant Branch (AGB) and mass loss phases to the white dwarf regime. Abundance changes are accounted for by means of a full coupling between nuclear evolution and timedependent mixing due to convection, salt fingers, and diffusive overshoot. In addition, time-dependent element diffusion for multicomponent gases has been considered during the white dwarf evolution. Crystallization and chemical rehomogenization due to phase separation upon crystallization in the core of our models have been fully considered. The effects of crystallization on the period spectrum of these massive white dwarf models are assessed by means of a detailed pulsational analysis of linear, nonradial, adiabatic gravity modes. To properly account for the effects of the presence of a solid phase in the models we impose special conditions on the oscillation eigenfunctions at the solid-liquid interface. We find that the theoretical pulsation spectrum is strongly modified when crystallization is considered, in particular concerning the mode trapping properties of the equilibrium models. We show that the strong mode trapping seen in the models with overshooting can be reproduced by means of a simple analytical model. We also discuss at some length the implications of our study for BPM 37093, the most massive ZZ Ceti star presently known. In particular, we attempt to place constraints on the physical processes occurring prior to the formation of this white dwarf. We find that if BPM 37093 has a stellar mass of $\approx 1.00 M_{\odot}$ its observed spectrum could bear the signature of overshoot episodes during the helium core burning.
\end{abstract}

Key words. dense matter - stars: evolution - stars: white dwarfs - stars: oscillations

\section{Introduction}

ZZ Ceti (or DAV) stars are cool, hydrogen-rich pulsating white dwarf stars belonging to the currently most extensively studied family of degenerate pulsators - see Gautschy \& Saio $(1995,1996)$ for a review. Their pulsating nature is evident

^ Member of the Carrera del Investigador Científico y Tecnológico, CONICET, Argentina. from periodic brightness variations ${ }^{1}$ caused by spheroidal, nonradial $g$ (gravity)-modes of low degree $(\ell \leq 2)$. Excitation of $100-1200 \mathrm{~s}$ oscillation modes is likely to be due to the action of the so called convective driving mechanism (Brickhill 1991; Goldreich \& Wu 1999), although the early works of Dolez \& Vauclair (1981) and Winget et al. (1982) postulated

\footnotetext{
${ }^{1}$ In some ZZ Ceti stars, fluctuations have also been measured recently in radial velocity; see van Kerkwijk et al. (2000) and Kotak et al. (2002).
} 
the $\kappa-\gamma$ mechanism as responsible for driving. ZZ Ceti stars are well known to pulsate in a "pure" instability strip at effective temperatures $\left(T_{\text {eff }}\right)$ between about 11000 and $12400 \mathrm{~K}$. Relevant studies devoted to exploring the pulsational properties of ZZ Ceti stars are those of Brassard et al. (1991b, 1992a,b), Gautschy et al. (1996), Bradley (1996, 1998a, 2001) and Córsico et al. (2001, 2002) amongst others.

Over the last years, pulsation studies of ZZ Ceti stars - as well as of DBV, DOV and PNNV stars, the other categories of pulsating white dwarfs - through asteroseismology have become a valuable technique for sounding the white dwarf interiors and evolution. Indeed, asteroseismological inferences have provided independent valuable constraints on fundamental quantities such as core composition, outer layer chemical stratification and stellar mass (Pfeiffer et al. 1996; Bradley 1998b, 2001).

A prominent application of white dwarf asteroseismology that has drawn the attention of researchers is related to the possibility of placing observational constraints on the crystallization process in the very dense interiors of white dwarfs. Crystallization in the core of white dwarfs was predicted theoretically about 40 years ago (Van Horn 1968; see also Kirzhnits 1960; Abrikosov 1960; Salpeter 1961), but researchers have not been able to peer into the crystallized structure of white dwarfs until recently. This has been prompted by the discovery of pulsations in the star BPM 37093 (Kanaan et al. 1992), a massive ZZ Ceti star which should be largely crystallized (Winget el al. 1997). In a very detailed investigation, Montgomery \& Winget (1999) explored the effects of crystallization on the period pattern of massive white dwarf stellar models. Amongst other findings, these authors concluded that some periods are notoriously sensitive to changes in the crystallized mass fraction of their models. They concluded that pulsating white dwarfs are, in principle, very promising objects to place constraints on the crystallization processes in stars.

Very recently, Metcalfe et al. (2004) have performed asteroseismological fits to the pulsation periods of the star BPM 37093. In their models, the crystallized mass fraction was treated as a free parameter, with the goal of empirically determining the degree of crystallization for a given stellar mass and core composition. Their preliminary results are that BPM 37093 has a crystallized mass fraction greater than $50 \%$, with values possibly as high as $90 \%$, although they indicated that an exploration of a finer grid of models (in both stellar mass and crystallized mass fraction) will be necessary in order to make conclusive statements.

Crystallization has consequences for the carbon/oxygen distribution within the core of a white dwarf. As a matter of fact, solid theoretical evidence suggests that, when crystallization occurs, the oxygen content in the solid phase is enhanced relative to that in the original fluid phase (Stevenson 1980; García-Berro et al. 1988; Ichimaru et al. 1988; Segretain \& Chabrier 1993). As a result, the carbon content in the fluid surrounding the solid core is enhanced. Since carbon is lighter than oxygen, these fluid layers are Rayleigh-Taylor unstable, and the ensuing convective mixing will redistribute the abundances and lead to flat profiles in a region whose size depends on the initial composition profile and on the degree of chemical enhancement produced during the crystallization process (Isern et al. 1997; Salaris et al. 1997; Montgomery et al. 1999). These mixing episodes could be particularly relevant in the context of carbon/oxygen cores with varying chemical profiles, since in principle they could be smoothed out by such chemical rehomogenization. To the best of our knowledge, pulsational studies of massive white dwarfs taking into account the chemical rehomogenization induced by phase separation have not been performed; the effect was not considered in the pulsational calculations of Montgomery \& Winget (1999), although it was fully taken into account in the calculations of phase separation and cooling delay in white dwarfs by Salaris et al. (1997), Montgomery et al. (1999), and Salaris et al. (2000). In view of these considerations, one of the main motivations for the present paper concerns the full exploration of the pulsational properties of massive white dwarfs that takes into account the effects of phase separation in carbon/oxygen cores.

Recently, Althaus et al. (2003) - hereinafter Paper I - have presented new stellar models for massive ZZ Ceti stars, based on detailed evolutionary calculations that account for the complete evolution of the white dwarf progenitor. In particular, such calculations include diffusive mechanical overshooting during the core burning phases of the progenitor star. In addition, the evolution of the chemical abundance distribution due to time-dependent element diffusion during the whole white dwarf regime has been considered in Paper I. In that work we concluded that, as a result of the smoothness of the chemical profile caused by diffusion processes, the mode trapping due to outer chemical interfaces is notably diminished, irrespective of the occurrence of core overshooting. Instead, the theoretical pulsational spectrum is characterized by the presence of pronounced non-uniformity in the spacing of consecutive periods in models with core overshooting, at variance with the situation in which this mixing process is neglected. In particular, we found that the pulsational spectrum in models with overshooting is dominated by the presence of "core trapped" modes, characterized by relatively high values of the oscillation kinetic energy and strong minima in the period spacing diagrams.

The main conclusion drawn in Paper I is that the pulsational properties of massive $\mathrm{ZZ}$ Ceti stars become very sensitive to the occurrence of extra mixing episodes that take place beyond the formally convective core during the central helium burning, for instance core overshoot. Such mixing episodes give rise to a sharp variation of the core chemical composition that leaves noticeable signatures on the theoretical period spectrum of pulsating white dwarfs. However, the fact that the eigenfunctions of $g$-modes are expected to have very low amplitudes in the solid core - because of the non-zero shear modulus of the solid (Montgomery \& Winget 1999) - and the mixing episodes induced by crystallization were two effects not addressed in Paper I. Therefore, an initial question to be answered in this paper is whether the chemical discontinuity caused by core overshoot could be wiped out by the chemical rehomogenization, and more importantly, what implications this would have for the pulsational properties of massive ZZ Ceti stars. In this connection, another aim of our work is to place constraints on the 
stellar mass and $T_{\text {eff }}$ values at which we should expect a pulsational pattern without any signature of core overshoot.

We believe that a re-examination of the pulsational properties of massive white dwarf stars deserves to be done in the frame of our new massive ZZ Ceti model stars. Specifically, our aim is to explore the pulsational properties of the white dwarf models presented in Paper I taking crystallization selfconsistently into account. Section 2 contains a brief description of the main physical inputs of the models. In Sect. 3 we explore the response of the pulsational pattern to the presence of crystallization. Section 4 is devoted to discussing the implications of our results for observational expectations and, finally, in Sect. 5 we make some concluding remarks.

\section{Input physics and evolutionary sequences}

Our massive, carbon/oxygen-core white dwarf models appropriate for ZZ Ceti stars have been obtained with the LPCODE evolutionary code described at length in Paper I and references therein. The code is based on an up-to-date and detailed physical description, particularly regarding the processes responsible for the chemical changes. In this section we summarize the input physics of our stellar code and the main characteristics of our evolutionary models.

LPCODE uses OPAL radiative opacities - including carbon- and oxygen-rich compositions - for arbitrary metallicity from Iglesias \& Rogers (1996) and molecular opacities from Alexander \& Ferguson (1994). The equation of state includes partial ionization, radiation pressure, ionic contributions, partially degenerate electrons and Coulomb interactions. For the white dwarf regime we include an updated version of the equation of state of Magni \& Mazzitelli (1979). Neutrino emission rates and high-density conductive opacities are taken from the works of Itoh and collaborators - see Althaus et al. (2002). A total of 34 thermonuclear reaction rates and 16 isotopes characterizes our nuclear network that describes the hydrogen - proton-proton chain and CNO bi-cycle - and helium burning, and carbon ignition. Nuclear reaction rates are taken from Caughlan \& Fowler (1988) and Angulo et al. (1999).

Concerning the abundance changes, we consider a timedependent scheme for the simultaneous treatment of chemical changes caused by nuclear burning and mixing processes. Specifically, the changes in the abundances for all chemical elements are described by the set of equations

$\left(\frac{\mathrm{d} \boldsymbol{Y}}{\mathrm{d} t}\right)=\left(\frac{\partial \boldsymbol{Y}}{\partial t}\right)_{\mathrm{nuc}}+\frac{\partial}{\partial M_{r}}\left[\left(4 \pi r^{2} \rho\right)^{2} D \frac{\partial \boldsymbol{Y}}{\partial M_{r}}\right]$,

with $\boldsymbol{Y}$ being the vector containing the number fraction of all considered nuclear species. Here, mixing due to convection, salt finger and overshoot is treated as a diffusion process which is described by the second term of Eq. (1). The efficiency of convective and salt-finger mixing is described by appropriate diffusion coefficients, $D$, which are specified by the treatment of convection. In particular, we considered the extended mixing length theory of convection for fluids with composition gradients developed by Grossman et al. (1993) in its local approximation as given by Grossman \& Taam (1996).
This treatment applies in convective, semiconvective ${ }^{2}$ and salt finger instability regimes. The first term of Eq. (1) gives the abundance changes due to thermonuclear reactions, changes which are fully coupled to mixing processes. This term is linearized following the implicit scheme of Arnett \& Truran (1969). For details concerning the numerical scheme employed to solve Eq. (1) see Paper I.

The evolution of the chemical abundance distribution caused by diffusion processes during the whole white dwarf regime has been taken into account in this work. Our timedependent element diffusion treatment, based on the formulation for multicomponent gases presented by Burgers (1969), considers gravitational settling, and chemical and thermal diffusion ${ }^{3}$ for the nuclear species ${ }^{1} \mathrm{H},{ }^{3} \mathrm{He},{ }^{4} \mathrm{He},{ }^{12} \mathrm{C}$, and ${ }^{16} \mathrm{O}$. In this way, we avoid the use of the trace element approximation invoked in most ZZ Ceti studies.

Concerning overshooting, we have included timedependent overshoot mixing during all pre-white dwarf evolutionary stages. We have considered exponentially decaying diffusive overshooting above and below any formally convective region, including the convective core (main sequence and central helium burning phases), the external convective envelope and the short-lived helium-flash convection zone which develops during the thermal pulses. Specifically, we have followed the formalism of Herwig (2000).

The effect of crystallization on the pulsational pattern of ZZ Ceti stars constitutes the central point of this paper. For a white dwarf model characterized by a given $T_{\text {eff }}$ value, stellar mass, and chemical composition, LPCODE provides a formally self-consistent value of the crystallized mass fraction $\left(M_{\mathrm{c}} / M_{*}\right)$. In our calculations, crystallization is assumed to occur when $\Gamma>180$ (Ogata \& Ichimaru 1987; Stringfellow et al. 1990), where $\Gamma \equiv Z^{2} e^{2} / \bar{r} k_{\mathrm{B}} T$ is the ion coupling constant. In order to evaluate self-consistently the perturbations caused by a crystal/fluid interface on the eigenfunctions we shall adopt special boundary conditions (see Sect. 3.1). In addition, the chemical redistribution due to phase separation has been taken into account following the procedure described in Montgomery et al. (1999) and Salaris et al. (1997). To assess the enhancement of oxygen in the crystallizing core we have employed the phase diagram of Segretain \& Chabrier (1993). Details concerning the algorithm used to compute the mixing processes due to crystallization will be described in Sect. 3.5 below.

An important aspect of the present study is to explore the implications of the occurrence of overshooting during the prewhite dwarf evolution for the pulsational properties of crystallized ZZ Ceti stars. To this end we consider the evolutionary sequences analyzed in Paper I, that is sequence NOV based on the complete evolution of a 7.5- $M_{\odot}$ initial mass star in which overshooting was not considered, and sequence OV based on the evolution of a 6- $M_{\odot}$ star with overshooting (a solarlike initial composition has been adopted). For the two sequences the mass of the resulting carbon/oxygen core is quite

\footnotetext{
2 Semiconvective mixing - see Straniero et al. (2003) - has not been considered in our models.

3 Radiative levitation has been neglected.
} 
similar $\left(\approx 0.94 M_{\odot}\right)$. This has enabled us to compare the oscillation properties of crystallized ZZ Ceti stars characterized by the same stellar mass but being the result of the evolution of progenitor stars with different initial masses. It is worth mentioning that the $\mathrm{ZZ}$ Ceti models we employed in our analysis are the result of the complete evolution of massive white dwarf progenitors from the zero-age main sequence through the thermally pulsing and mass loss phases to the white dwarf regime. In particular, sequence OV experiences the third dredge-up and hot bottom burning during the thermally pulsing phase on the asymptotic giant branch (AGB). For details concerning the evolutionary properties of our sequences we refer the reader to Paper I. In what follows we restrict ourselves to commenting on the main results for the inner chemical composition.

The upper panels of Figs. 1 and 2 show the chemical profiles at the ZZ Ceti stage for sequences NOV and OV. As demonstrated in Paper I, except for the inner part of the core, where the diffusion time scale becomes much longer than the evolutionary time scale, element diffusion is so efficient that the resulting abundance distribution at the ZZ Ceti stage does not depend on the occurrence of overshooting in the convective envelope during the thermally pulsing AGB phase. In fact, the resulting external chemical profile at the ZZ Ceti stage is quite similar for the two sequences. Remarkably enough, our models are characterized by a chemical interface in which helium, carbon and oxygen in non-negligible abundances coexist, an interface which, when the white dwarf reaches the ZZ Ceti stage, has extended appreciably as a result of chemical diffusion. Needless to say, the trace element approximation would not be the appropriate approach to treat element diffusion.

However, overshoot episodes occurring during the core burning phases, particularly during the helium core burning, leave recognizable features in the inner chemical profile of a massive ZZ Ceti star, as is documented by Fig. 2. That is, the innermost region of such stars keeps a record of the extra mixing experienced during the pre-white dwarf evolution. In particular, note the sharp variation of the carbon/oxygen profile at $\log q \approx-0.7\left(q=1-M_{r} / M_{*}\right)$. As shown in Paper I, this is responsible for noticeable structure in the period spacing diagrams. The shape of the chemical profile towards the central region of our OV model sequence is typical for situations in which additional mixing beyond what is predicted by Schwarzschild criterion for convective stability is allowed. The occurrence of such mixing episodes, particularly core overshooting and/or semiconvection, is suggested by both theoretical and observational evidence. In particular, extra mixing episodes beyond the external border of the fully convective core that take place towards the end of central helium burning have a large influence on the carbon and oxygen distribution in the core of white dwarfs. Recently, Straniero et al. (2003) presented a detailed analysis of the inner chemical abundance in a $3-M_{\odot}$ star model resulting from different extra mixing processes during the late stage of the core helium burning phase. In particular, they conclude that models which incorporate semiconvection or a moderate mechanical overshoot applied to core and convective shells predict a sharp variation of the chemical composition in the carbon/oxygen core, in agreement with our results for the OV sequence.

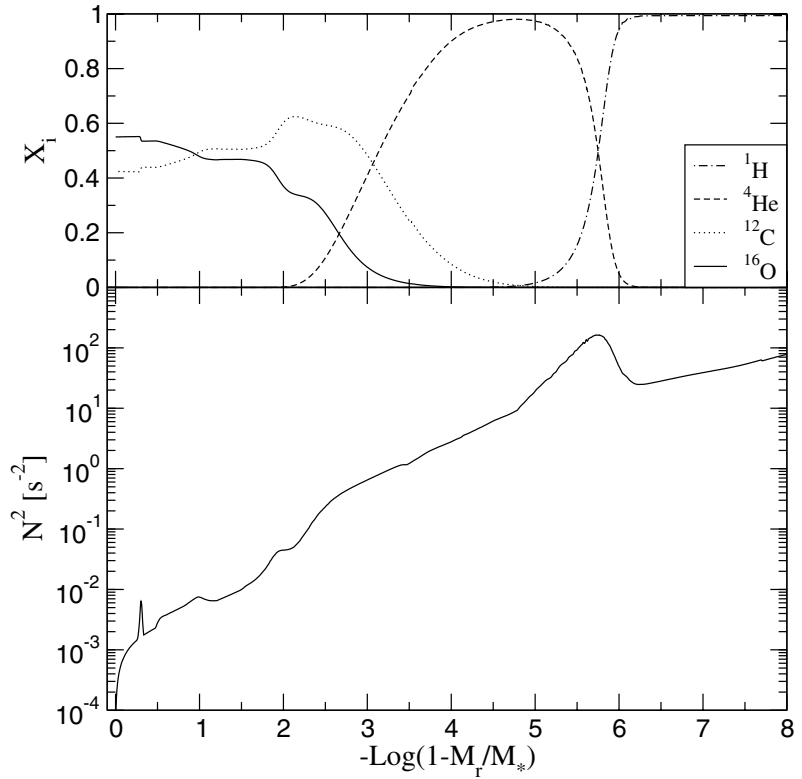

Fig. 1. Abundance by mass of ${ }^{1} \mathrm{H},{ }^{4} \mathrm{He},{ }^{12} \mathrm{C}$ and ${ }^{16} \mathrm{O}$ (upper panel) and the run of the Brunt-Väisälä frequency (lower panel) as a function of the outer mass fraction $q$ for the $0.936-M_{\odot}$ white dwarf remnant of 7.5- $M_{\odot}$ evolution (sequence NOV) near the ZZ Ceti instability strip. The model is characterized by $\log \left(L / L_{\odot}\right)=-2.92$ and $\log T_{\text {eff }}=4.06$.

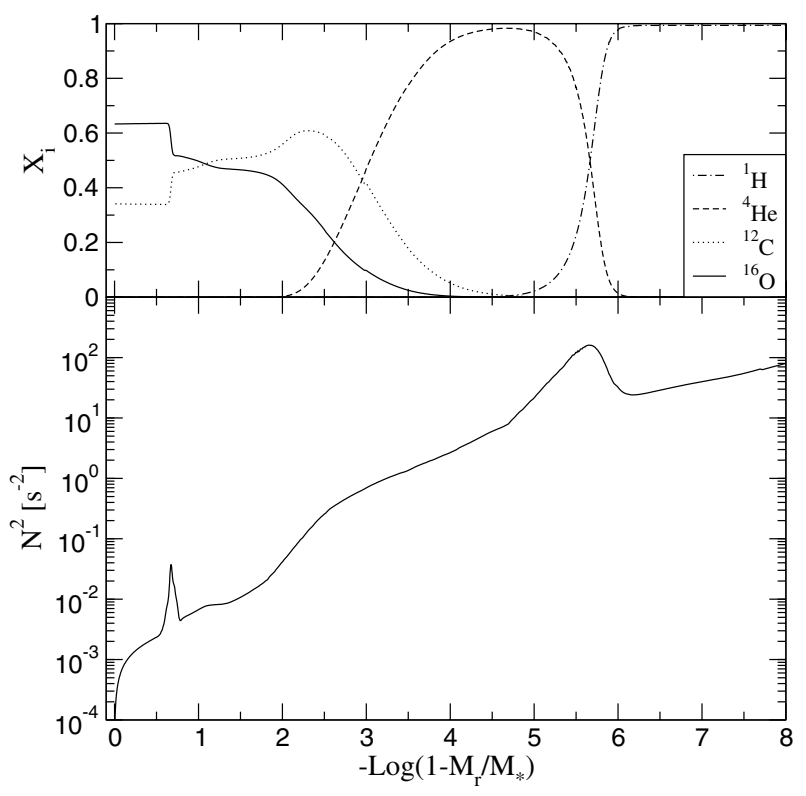

Fig. 2. Same as Fig. 1 but for the $0.94-M_{\odot}$ white dwarf remnant of $6-M_{\odot}$ evolution (sequence OV). Core overshooting leads to a inner chemical profile quite different from the situation in which no extra mixing beyond the fully convective core is considered (Fig. 1). In particular note the almost-discontinuity in the chemical profile at $\log \left(1-M_{r} / M_{*}\right) \approx-0.7$.

\section{Pulsational analysis}

In this section we shall explore in detail the consequences for the theoretical period spectrum of our white dwarf models due to the effects of crystallization. In all the cases we shall analyze model-sequences NOV and OV in models with $T_{\text {eff }}$ decreasing from $\approx 15000$ to $\approx 10000 \mathrm{~K}$, which covers the temperature 
range for the observed ZZ Ceti instability strip. In showing our results we shall concentrate on $\ell=2 g$-modes only; the results for other values of $\ell$ are qualitatively similar.

Before discussing the pulsational results, we briefly describe below the pulsational code and the treatment employed to assess the various pulsational quantities.

\subsection{Pulsation code}

For computing adiabatic, nonradial $g$-modes of the ZZ Ceti models we employ the same pulsational code as in Córsico et al. (2001, 2002), with appropriate modifications for handling the effects of crystallization on oscillation eigenmodes. Briefly, the code, which is coupled to the LPCODE evolutionary code, is based on the general Newton-Raphson technique to solve the full set of linearized equations governing spheroidal ${ }^{4}$, adiabatic, nonradial pulsations of spherically symmetric stars. The code provides the eigenperiod $P_{k}$ ( $k$ being the radial overtone of the mode) and the dimensionless eigenfunctions $y_{1}, \cdots, y_{4}$ - see Unno et al. (1989) for their definition. Useful pulsation quantities, such as the oscillation kinetic energy, the weight function, and the variational period - see Kawaler et al. (1985) - are also provided by our pulsational code for each computed eigenmode. Finally, the asymptotic period spacing is computed as in Tassoul et al. (1990).

The boundary conditions are those given by Osaki \& Hansen (1973) and the normalization condition adopted at the stellar surface is $y_{1}=1$. Concerning the inner boundary conditions of our crystallizing models, we have adopted the "hard-sphere" boundary condition, which has been shown by Montgomery \& Winget (1999) to be a realistic representation. In fact, the amplitude of eigenfunctions of $g$-modes is drastically reduced below the solid/liquid interface due to the nonzero shear modulus of the solid, as compared with the amplitude in the fluid region - see Fig. 4 of Montgomery \& Winget (1999).

Specifically, the hard-sphere boundary condition at the radial shell corresponding to the outward-moving crystallization front $\left(r_{\mathrm{c}}=r\left(M_{\mathrm{c}}\right)\right)$ reads

$$
\begin{aligned}
& y_{1}=0, \\
& y_{2}=\text { arbitrary, } \\
& \ell y_{3}-y_{4}=0 .
\end{aligned}
$$

Here $y_{1}$ and $y_{2}$ represent the radial and tangential displacements, respectively, and $y_{3}$ and $y_{4}$ are the Eulerian perturbation of the gravitational potential and its derivative. Note that the last condition is the same as for the normal case in which the core is in a fluid state and the boundary condition is applied at the stellar centre - see Appendix B of Montgomery \& Winget (1999).

\footnotetext{
4 We have not considered torsional modes, since these modes are characterized by very short periods - up to $20 \mathrm{~s}$; see Montgomery \& Winget (1999) - and are not observed in ZZ Ceti stars.
}

\subsection{The Brunt-Väisälä frequency}

The procedure we follow to assess the run of the Brunt-Väisälä frequency $(N)$ is that proposed by Brassard et al. (1991b). This numerical treatment takes explicit account of the contribution to $N$ from any change in composition in the white dwarf models by means of the Ledoux term $B$. This is an important aspect in connection with the phenomenon of mode trapping and confinement (Brassard et al. 1992a; Córsico et al. 2002). In the lower panels of Figs. 1 and 2 we show the run of $N^{2}$ in terms of the outer mass fraction for white dwarf models at $T_{\text {eff }} \approx 11800 \mathrm{~K}$ corresponding to sequences NOV and OV, respectively. Element diffusion strongly smooths out the external chemical profile emerging from the thermally pulsing AGB phase to such an extent that the Brunt-Väisälä frequency in the outer layers exhibits very smooth local features. Note that because of the distinct shape of the chemical distribution in the innermost region of the model with core overshooting, the profile of $N$ for such a model exhibits a clearly peaked feature at $\log q \sim-0.7$ (Fig. 2). This feature is responsible for the presence of pronounced minima in the period spacing distribution (see Fig. 15 of Paper I). The model corresponding to sequence NOV also shows an innermost peak in $N$ (Fig. 1). However, because it is located at a deeper layer and has a smaller amplitude than in the OV sequence, it produces a much smaller amount of mode trapping.

\subsection{Results without crystallization}

In this section we shall describe pulsational results in which we have neglected crystallization in the computation of the pulsational spectrum. In Fig. 3 we show the evolution of the $\ell=2$ periods in terms of $T_{\text {eff }}$ corresponding to the NOV sequence of models. Clearly, the periods increase monotonically with decreasing $T_{\text {eff }}$, particularly in the case of modes with high radial order $k$. This effect is explained on the basis that the Brunt-Väisälä frequency at the core decreases as a result of increasing degeneracy in the core. Note that the mode with $k=1$ is rather insensitive to the white dwarf cooling, though its period slightly grows with decreasing $T_{\text {eff. }}$. We note that the curves are very smooth in the whole $T_{\text {eff }}$ range considered, and only in a few cases is there a slight approach between periods of adjacent modes. In Fig. 4 we depict the evolution of periods in the case of models corresponding to the OV sequence. In this case the overall trend of the periods is similar to that found in NOV models, but they show instead clear signals of mode bumping and avoided crossing ${ }^{5}$. For instance, a series of mode bumping is observed between modes with $k=17$ and $k=18, k=18$ and $k=19, k=19$ and $k=20$, and $k=20$ and $k=21$ (thick lines in Fig. 4). We also find that, in addition to the periods, the kinetic energy and period spacing minima are also exchanged between these pairs of modes after mode bumping has taken place. A close inspection of the eigenfunctions and weight functions demonstrates that modes experiencing avoided crossings are core trapped ones, that is, modes

\footnotetext{
5 When a pair of modes experiences avoided crossing, the modes exchange their intrinsic properties after mode bumping; see Aizenman et al. (1977).
} 


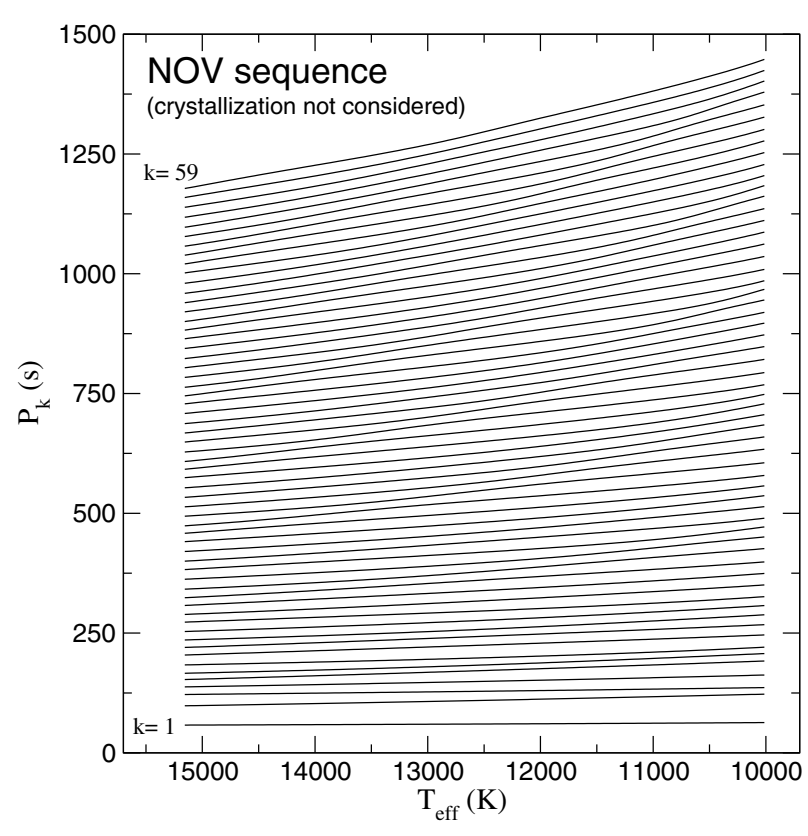

Fig. 3. Evolution of the periods for $\ell=2$ modes as a function of the effective temperature, corresponding to the NOV model sequence. The effects of crystallization have been neglected in computing the eigenmodes.

with relatively large amplitudes in the region bounded by the centre of the model and the location of the step in the oxygen profile left by overshooting (see Paper I). Similar mode bumping/avoided crossing features in white dwarf evolutionary models have been reported by Wood \& Winget (1988) and Brassard et al. (1991a), although in such calculations modes experiencing avoided crossings were identified as modes trapped in the outer hydrogen envelope. In the context of massive white dwarf models, Montgomery \& Winget (1999) have found avoided crossing phenomena when the crystallized mass fraction of their models is varied in a continuous fashion and the other model parameters (such as $T_{\text {eff }}$ ) are held fixed.

\subsection{Results considering crystallization without chemical rehomogenization}

In this section, we describe our results when account is taken of the presence of a solid core in the computation of the pulsational eigenspectra. However, we neglect here the effect of chemical rehomogenization due to phase separation, that is, the core chemical profile remains fixed as the white dwarf crystallizes. We stress that we take the crystallized mass fraction not as a free parameter but as given by evolutionary computations. Figures 5 and 6 depict the resulting evolution of periods in terms of $T_{\text {eff }}$ for sequences NOV and OV, respectively. Note that in both cases the onset of crystallization is reflected in the pulsational spectrum as a rather abrupt increase in periods for modes with high- and intermediate radial orders, whereas lower overtones are rather insensitive to the presence of the solid core. This is because for $g$-modes, when $k \gg 1$ (asymptotic limit), the periods are given by $P_{k} \propto k\left(\int_{r_{1}}^{r_{2}} \mathrm{Nd} r / r\right)^{-1}$ (Tassoul et al. 1990), where $r_{1}$ and $r_{2}$ define the propagation

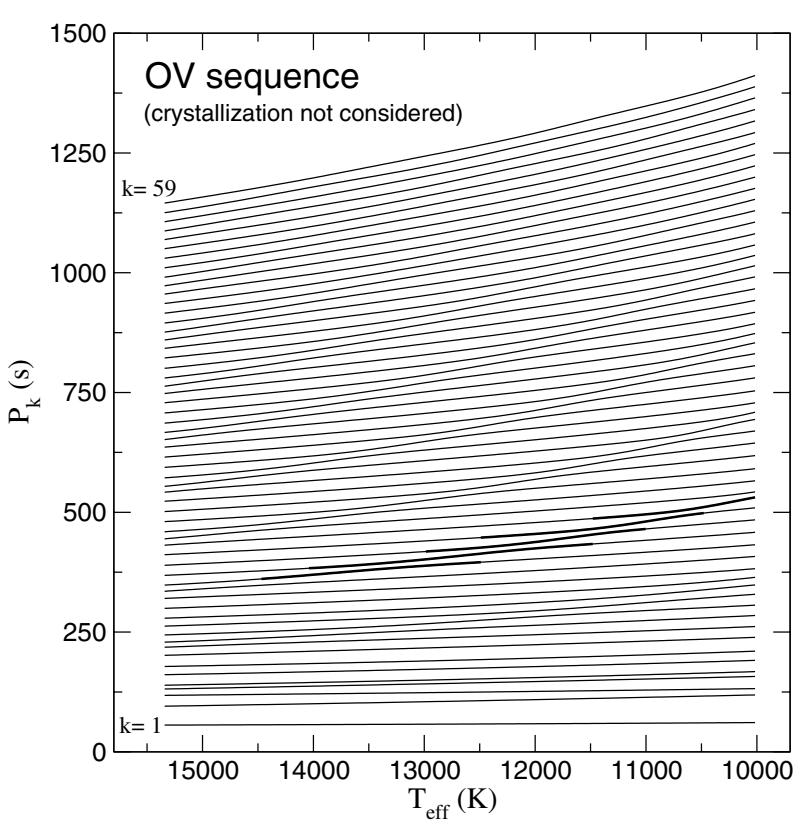

Fig. 4. Same as Fig. 3, but for the OV model sequence. Thick lines emphasize some examples of modes experiencing bumping/avoided crossing phenomena.

region of modes. When crystallization is considered, the internal boundary $r_{1}\left(M_{\mathrm{c}}\right)$ moves outward, so the integral $\int_{r_{1}}^{r_{2}} N \mathrm{~d} r / r$ decreases, and consequently the periods (and the period spacings) increase. An important feature displayed by Figs. 5 and 6 is that when crystallization begins the mode bumping/avoided crossing phenomena propagate to longer periods. Indeed, the outward-moving crystallization front reinforces the process of mode bumping/avoided crossing, which is also present before the onset of crystallization. This effect is particularly noticeable in the case of OV models. In Figs. 7 and 8 we show the period spacing diagrams corresponding to various percentages of the crystallized mass fraction for NOV and OV model sequences, respectively. Note that in the case of OV models the strong minima in the period spacing are gradually displaced to longer periods as crystallization proceeds. At the same time they move away from each other. Note that at $T_{\text {eff }} \approx 10000 \mathrm{~K}(40 \%$ of crystallized mass fraction) only one minimum at about $550 \mathrm{~s}$ remains. The period spacing diagrams exhibited by models with core overshooting are completely different from those of models in which overshooting is neglected, as can be inferred from Fig. 7. In fact, although the period spacing diagrams are strongly modified by crystallization, their overall structure looks very different between the two sequences. In particular, sequence NOV lacks the strong minima characterizing sequence OV, features which, as concluded in Paper I, could eventually be used to place constraints on the occurrence of core overshooting. So, the conclusions arrived at in Paper I concerning the role of core overshooting in massive ZZ Ceti stars remain valid even when the effect of a solid core on the pulsations is included. However, this comes with the caveat that if the star is more massive than this model, it could have a large enough crystallized mass fraction $(\sim 80 \%)$ so that the chemical step left by overshooting would be engulfed by the crystallizing 


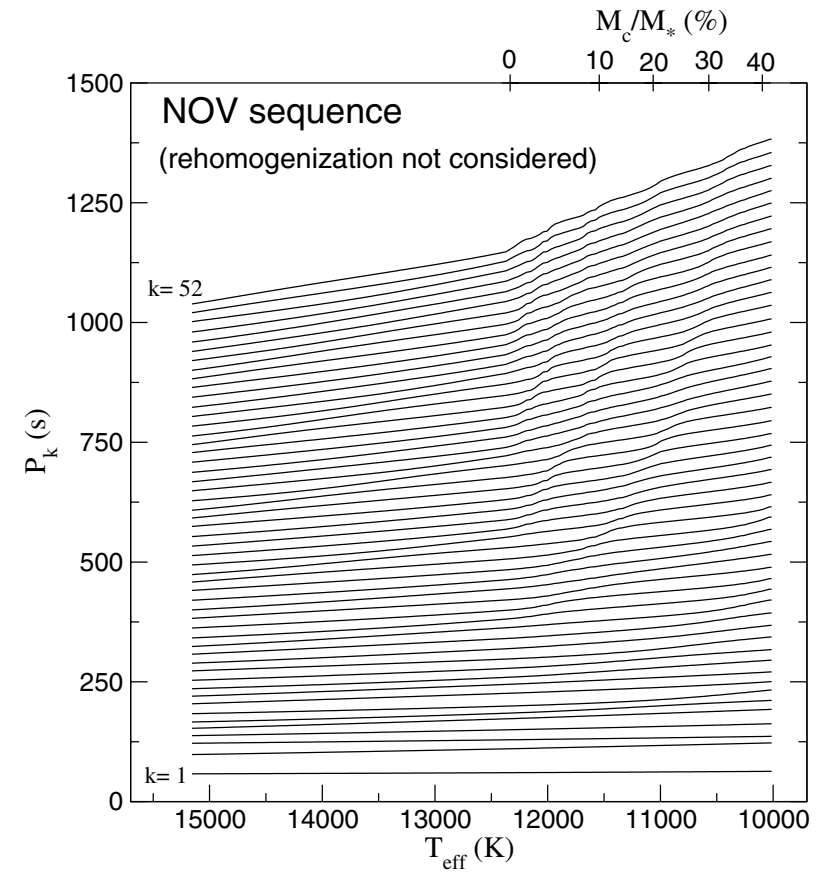

Fig. 5. Same as Fig. 3, but for the case in which the presence of a solid core has been considered in the computation of the eigenmodes. Chemical rehomogenization upon crystallization has not been taken into account in the stellar model. The upper scale measures the crystallized mass fraction.

core, erasing this feature. In this case we should expect a pulsational pattern with no appreciable minima in the period spacing. As we will see in the following section, this situation can be reached for smaller amounts of crystallization if the effects of phase separation and mixing are taken into account.

\subsection{Results considering crystallization and chemical rehomogenization}

As recently shown, the occurrence of core overshooting strongly modifies the appearance of the period diagrams even in the presence of a solid core. Now, theoretical evidence strongly suggests that if the white dwarf core is composed initially of a mixture of carbon and oxygen the crystallized region will have an enhanced abundance of oxygen compared to that in the original fluid state (Ichimaru et al. 1988; Segretain \& Chabrier 1993; Salaris et al. 1997). On the other hand, the fluid regions overlying the crystallized oxygen-enhanced layers will have a higher content of carbon. This region will become Rayleigh-Taylor unstable, since carbon is slightly less dense than oxygen. This instability leads to a rehomogenization of the chemical profile, and, as a result, the chemical abundances after crystallization can differ substantially when compared with the initial ones. This is an important point because any feature present in the chemical profile could be potentially wiped out by this rehomogenization process, even in fluid layers located far away from the crystallization front.

To compute the above-mentioned chemical rehomogenization, we have employed the same algorithm as in Montgomery et al. (1999) and Salaris et al. (1997). To derive the oxygen

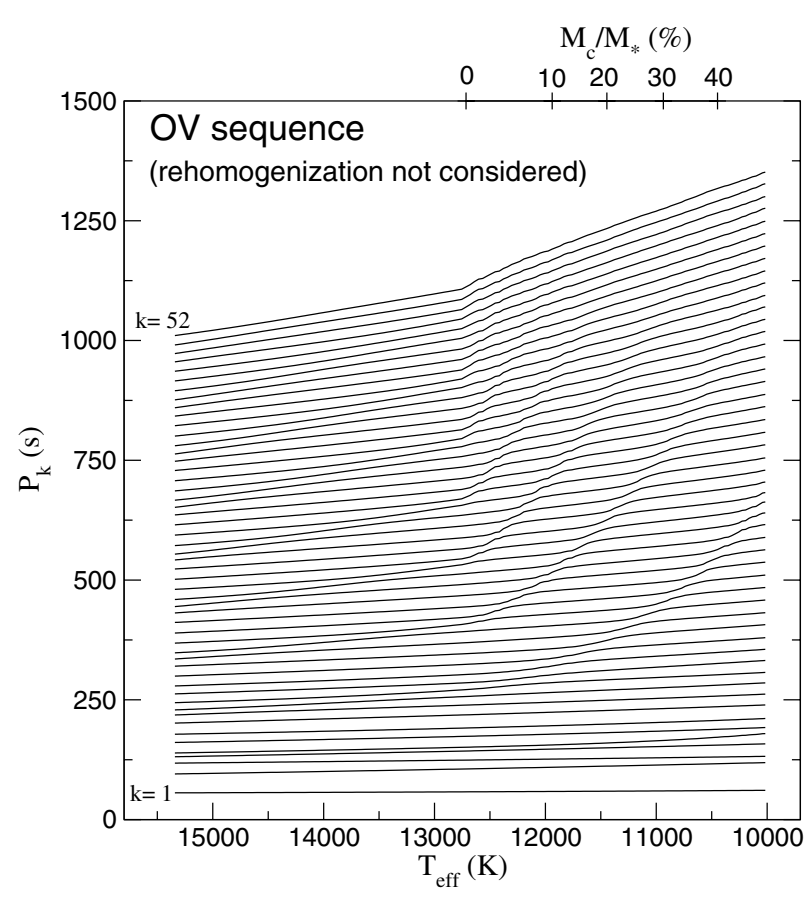

Fig. 6. Same as Fig. 5, but for the OV model sequence.

enhancement when a given layer crystallizes, we adopt the Segretain \& Chabrier (1993) spindle-type phase diagram for a carbon/oxygen mixture. In our scheme for mixing, we first consider a crystallized, oxygen-enhanced layer and then we ask if the innermost fluid shell has a higher carbon content than the overlying one. If it does, then we mix both fluid layers and perform the same comparison with the next layer further out. In this way we scan outward through the fluid and the process stops when further mixing no longer decreases the carbon content of the fluid between this point and the crystallization boundary. When the crystallization front moves outward due to cooling, the aforementioned procedure is repeated. In Fig. 9 we show the oxygen chemical profile in terms of the fractional mass for the OV model sequence corresponding to various degrees of crystallization. The initial, uncrystallized profile is depicted with a thick-solid line, and the resulting profiles after the rehomogenization has taken place are shown with thin lines for $10,20,30$ and $40 \%$ crystallized mass fractions. Note that the shape of the chemical profile is strongly modified, even in regions far from the crystallization front. As a clear example of this, we show by thick-dashed line the oxygen profile at the moment that the pronounced feature at $M_{r} \approx 0.80 M_{*}$ (left by core overshooting) is completely eliminated. This occurs when the location of the crystallization boundary reaches $M_{\mathrm{c}} \approx 0.37 M_{*}$.

We wish now to investigate how such chemical rehomogenization affects the pulsational spectrum of the white dwarf models. Figures 10 and 11 document the evolution of $\ell=2$ periods in terms of $T_{\text {eff }}$ corresponding to NOV and OV sequences respectively. For the case of the NOV sequence, the rehomogenization process induced by crystallization clearly modifies the internal chemical profile (it smooths out the profile at $\log q \approx-0.3$ ) to such a degree that any signal of mode bumping/avoided crossing is almost absent, in contrast with the situation in which such mixing has been neglected (see Fig. 5). 
NOV sequence, no rehomogenization

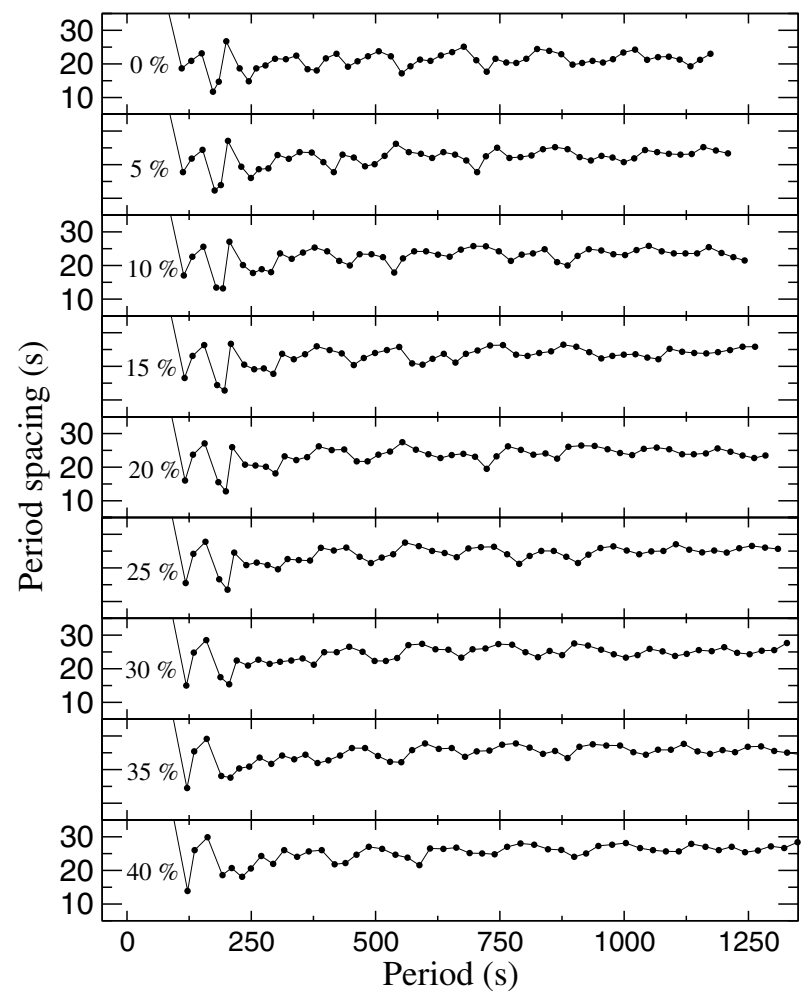

Fig. 7. The forward period spacing $\left(\Delta P_{k}=P_{k+1}-P_{k}\right)$ in terms of the periods for $\ell=2$ modes corresponding to the NOV model sequence. Each panel shows the predictions for different percentages of the crystallized mass fraction. Chemical rehomogenization was not considered.

For OV models, the theoretical spectrum of periods barely changes in response to the rehomogenization (compare Figs. 6 and 11). That is, clear features of mode bumping/avoided crossing survive even during the stages in which the pronounced step in the oxygen profile is continuously diminished by chemical rehomogenization (see Fig. 9). However, a closer inspection of Fig. 11 reveals that from $M_{\mathrm{c}} / M_{*} \approx 0.37\left(T_{\mathrm{eff}} \approx 10400 \mathrm{~K}\right)$ these features virtually disappear, and the period spacing becomes almost uniform. This effect can be appreciated more clearly from Fig. 13, in which we have plotted period spacing diagrams for several degrees of crystallization. Note that the pronounced minima characterizing the period spacing distribution at periods $\sim 760$ and $\sim 1120$ s (for $30 \%$ crystallization) are absent when the crystallized mass fraction is $35 \%$. In fact, when the chemical discontinuity has been completely wiped out by chemical rehomogenization, the predicted period spacing distribution becomes very flat, particularly for periods longer than $\sim 300 \mathrm{~s}$.

Figures 12 and 13 represent some of the main results of the present work. A comparison of these figures clearly indicates that the period spacing distribution for NOV and OV sequences is markedly different. In particular, we note that in the case of the OV model with $M_{\mathrm{c}}=0.35 M_{*}$, after the overshootinduced step has been almost removed from the chemical profile, the period spacing distribution is notably more uniform than in the NOV model characterized by the same percentage of
OV sequence, no rehomogenization

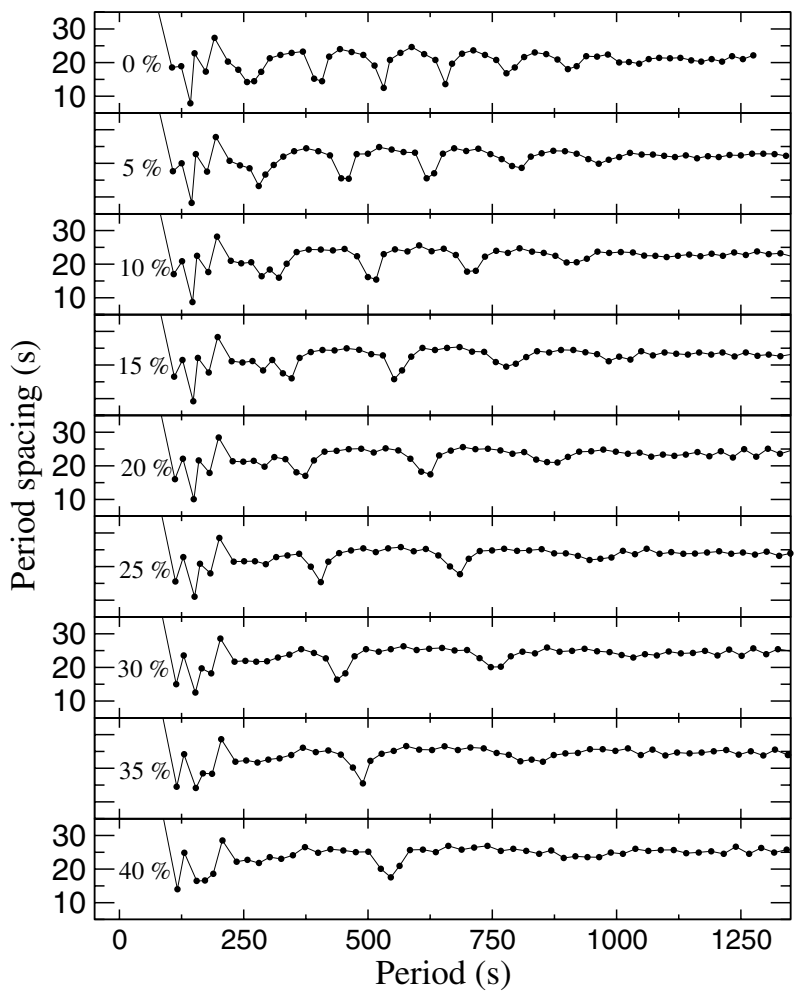

Fig. 8. Same as Fig. 7, but for the case of the OV model sequence.

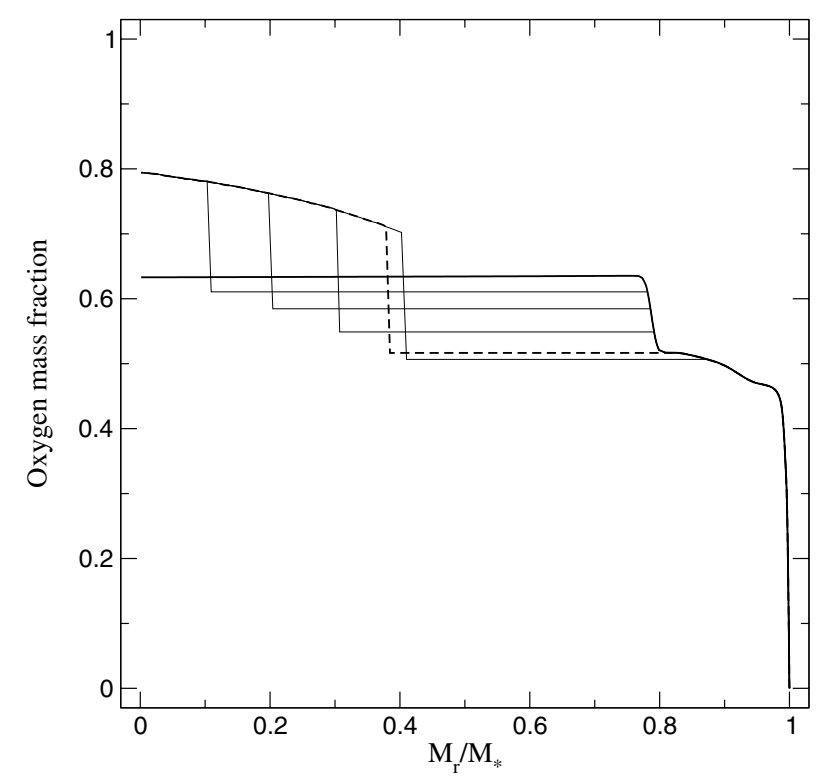

Fig. 9. Internal ${ }^{16} \mathrm{O}$ chemical profiles in terms of the fractional mass for the OV sequence corresponding to various percentages of crystallization. The thick-solid line corresponds to the profile just before the onset of crystallization, and thin lines correspond to the chemical profiles resulting from chemical rehomogenization at increasing percentages of crystallized mass fraction, from 10 to $40 \%$ in steps of $10 \%$. The curve at $40 \%$ of crystallized mass fraction corresponds to $T_{\text {eff }} \approx 10000 \mathrm{~K}$. The thick dashed line represents the oxygen profile by the time the chemical discontinuity left by core overshooting has been completely wiped out by chemical rehomogenization (when $\approx 37 \%$ of the core is in a crystallized state). 


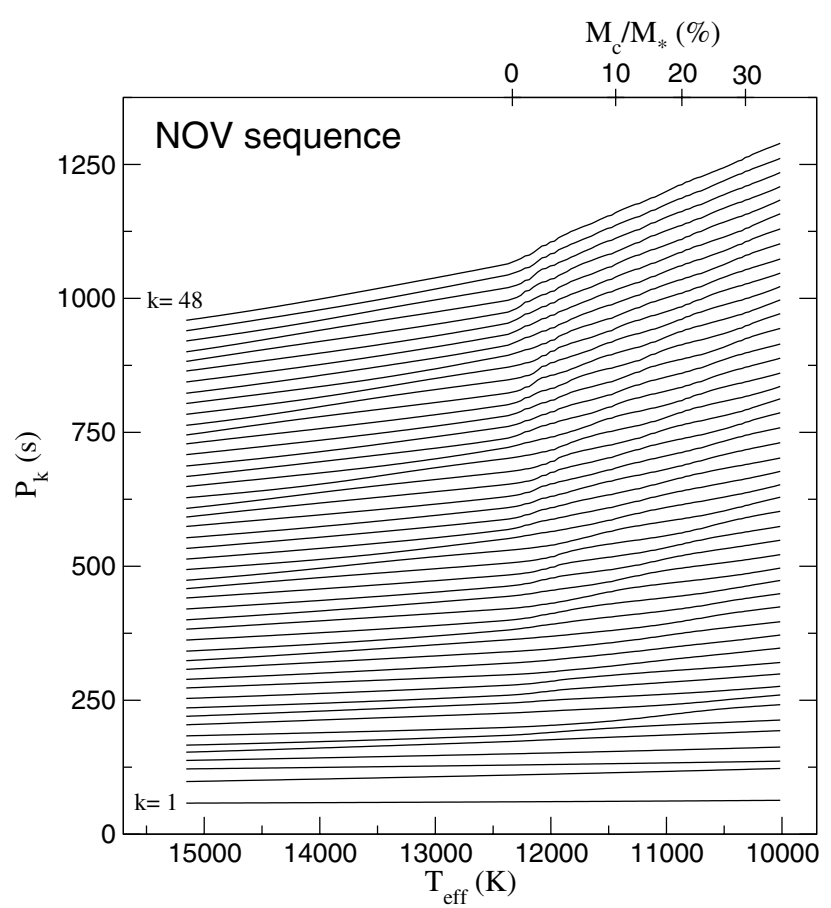

Fig. 10. Evolution of the periods for $\ell=2$ modes as a function of the effective temperature, corresponding to the NOV model sequence. The full effects of crystallization have been considered both in the assessment of the final shape of the internal chemical profile and in computing the eigenmodes.

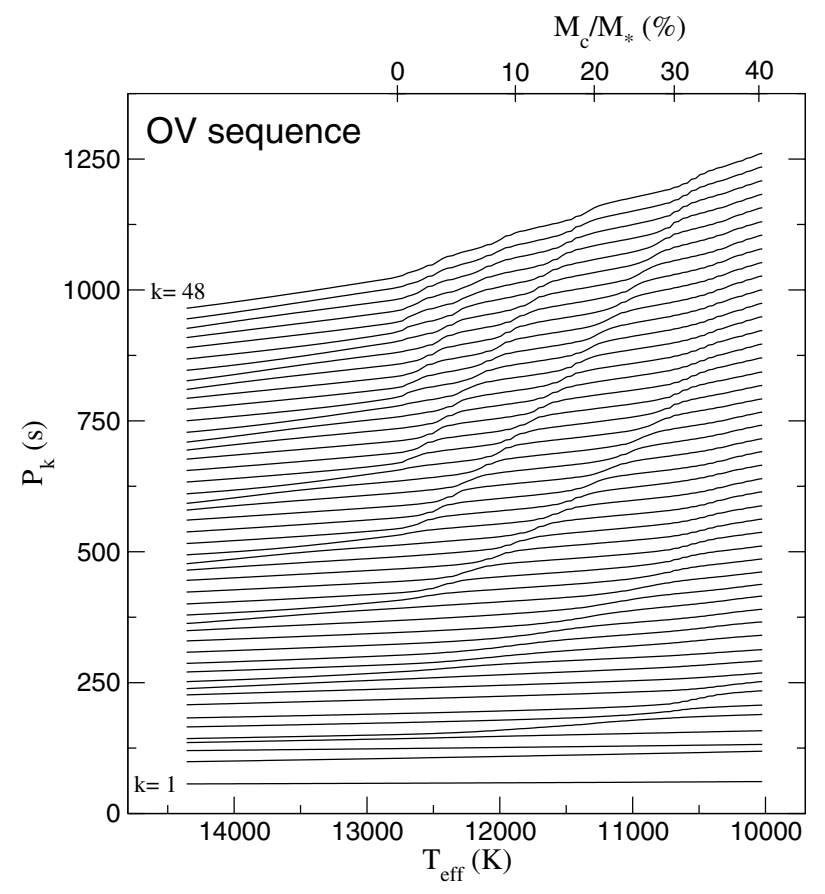

Fig. 11. Same of Fig. 10, but for the OV model sequence.

crystallization. This is because in regions farther out from the centre $(\log q \lesssim-1)$ the OV model is actually smoother than the NOV model, so its period spectrum shows less mode trapping.

Finally, in Figs. 14 and 15 we show the forward period spacing vs period for $15000 \mathrm{~K} \gtrsim T_{\text {eff }} \gtrsim 10000 \mathrm{~K}$ corresponding to the NOV and OV model-sequences, respectively. For the sake of clarity, each curve has been shifted upward starting from the lowest one, in increments of $1 \mathrm{~s}$. Labels of the solid curves indicate the percentage of crystallization and the corresponding $T_{\text {eff }}$ of the models. The dashed curve corresponds to the moment at which the relevant structure in the oxygen profile (at $\log q \approx-0.3$ for the NOV sequence, and at $\log q \approx-0.7$ for the OV sequence) disappears due to chemical rehomogenization. From these plots one can see the evolution of the trapped modes quite clearly, particularly when crystallization begins. Note from Fig. 15 the decrease in amplitude of mode trapping as the degree of crystallization increases. This figure also emphasizes the gradual change experienced by the period spacing when the step left by overshooting is removed by chemical rehomogenization.

\section{Analytical model of mode trapping}

In this section we examine the mode trapping in our models by comparing it to the results of a simple analytical model. This analytical model, which will be described in more detail elsewhere, describes the high-overtone limit of the pulsation equations as derived by Deubner \& Gough (1984) and Gough (1993). While formally equivalent to the standard linear adiabatic wave equation in the Cowling approximation - see, e.g., Cox (1980) - this form of the equations reduces the problem to a single second-order differential equation resembling the oscillations of a non-uniform string. In fact, using this equation as a convenient starting point, Montgomery et al. (2003) showed that a symmetry inherent in the string problem is still present in stellar pulsations. This led to the discovery of the core/envelope symmetry and the subsequent realization that the chemical composition gradients in the core may be even more effective in producing mode trapping than those in the envelope. Here we use an extended version of this model in which the perturbations to the background state need not be small, although our solutions are still formally valid only in the highovertone limit.

In Fig. 16 we illustrate how features/bumps in the BruntVäisälä frequency result in mode trapping in the eigenfunctions. As in Montgomery et al. (2003), we use the "normalized buoyancy radius", $\Phi$, defined by

$\Phi(r) \equiv \frac{\int_{0}^{r} \mathrm{~d} r \frac{|N|}{r}}{\int_{0}^{R_{\star}} \mathrm{d} r \frac{|N|}{r}}$,

as the radial variable, since with this choice the wavelength of the eigenfunctions appears to be constant, making the correspondence with the vibrating string more apparent ( $\Phi$ equals 0 and 1 correspond to the centre and surface of the model, respectively). In addition, the eigenfunction $\Psi \approx\left(r^{2} / g \rho^{1 / 2}\right) \delta p$ is defined in such a way that it has constant amplitude except in regions where the background state varies rapidly, such as the bumps shown in the top panel of Fig. 16. As can be seen, the "bump" at $\Phi \sim 0.16$ is narrower than the wavelengths of either the $k=5$ or $k=30$ mode, so both modes perceive it essentially as a delta function and therefore experience significant mode trapping (amplitude change) in propagating through it. In contrast, the other two "bumps" at $\Phi \sim 0.3$ and $\Phi \sim 0.6$ 
NOV sequence

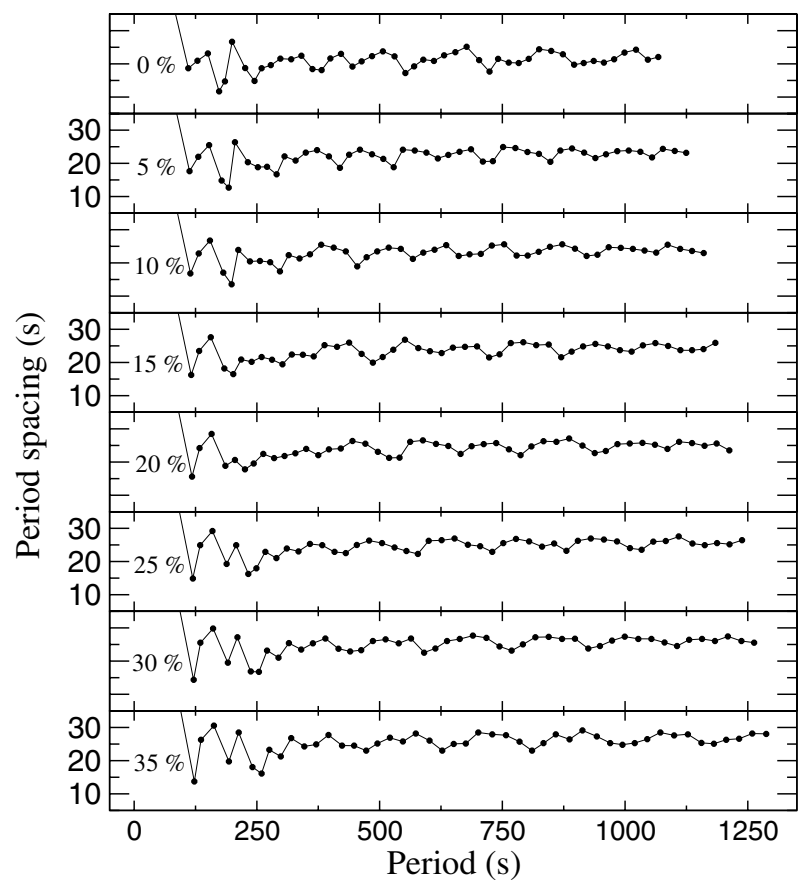

Fig. 12. The forward period spacing in terms of the periods for $\ell=$ 2 modes corresponding to the NOV model sequence analyzed in Fig. 10. Each panel shows the predictions for different percentages of the crystallized mass fraction. Here chemical rehomogenization induced by crystallization has been considered.

are much wider. Given that the wavelength of the $k=30$ mode is smaller than the width of these bumps, it experiences essentially no mode trapping. On the other hand, the wavelength of the $k=5$ mode is somewhat larger than the width of these bumps, so it still experiences a modest amount of mode trapping (amplitude change) due to these features.

In the top panel of Fig. 16 we have chosen the height, width, and position of the bumps in the analytical model (the solid curve) to mimic those found in a full evolutionary model (the dashed curve). In Fig. 17 we show the mode trapping found in our analytical model (filled circles, solid lines) and that found in the OV model shown in Fig. 13 (open circles, dotted lines), for values of the crystallized mass fraction between $0 \%$ and $40 \%$. Clearly, the overall correspondence is quite good, except for low periods, where our asymptotic model is not applicable anyway. In particular, the asymptotic model is able to reproduce i) the shape of the trapping cycle (sharp minima and flat maxima); ii) the decrease in amplitude of mode trapping for high periods/overtones; iii) the increase in the period of the trapping cycle and the movement of mode trapping features to higher periods as the degree of crystallization is increased; and iv) the absence of trapping features for the $40 \%$ crystallized case.

The explanation of these effects is straightforward. First, the overall good match is due to the fact that the modes shown actually have high overtone numbers and that the mode trapping is dominated by the single feature in $\delta N / N$ at $\Phi \sim$ 0.16 . Second, the decrease in trapping with increasing period

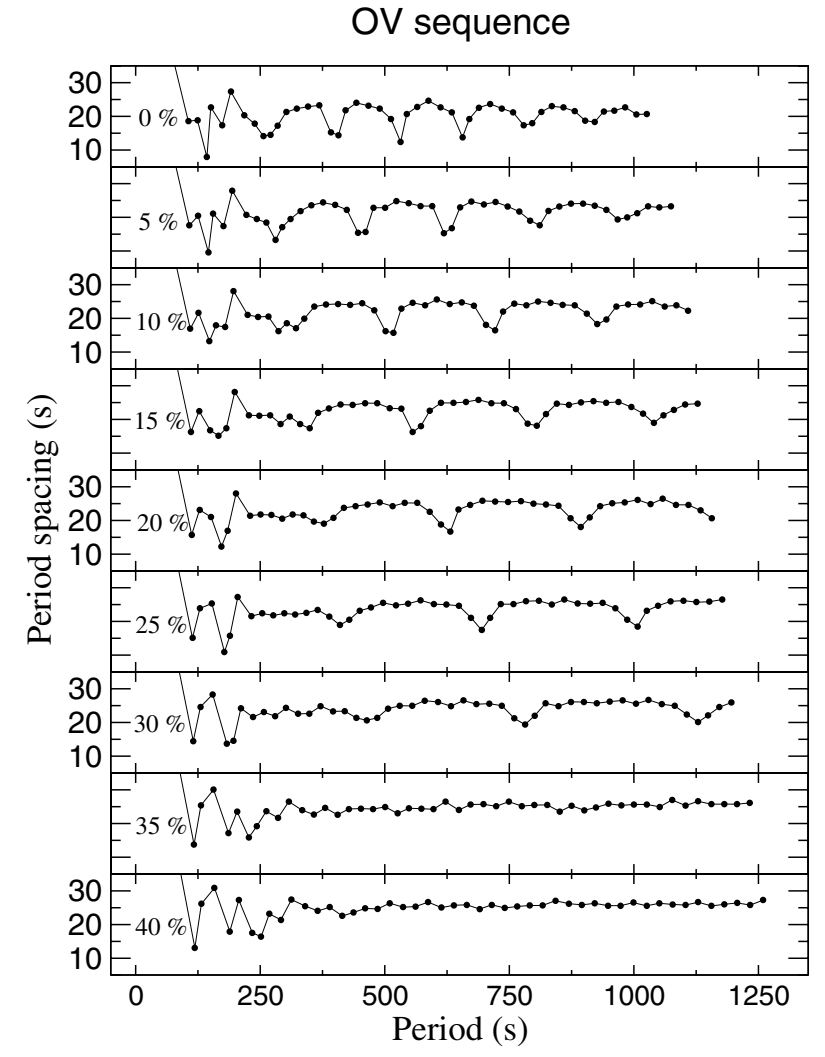

Fig. 13. Same as Fig. 12, but for the OV model sequence.

occurs because these modes have shorter spatial wavelengths, and when these wavelengths become comparable to the width of the features in $\delta N / N$ the modes cease to feel the effects of a sudden transition. Third, the reason that the number of modes per mode trapping cycle increases is that crystallization effectively moves the $\Phi=0$ point to the right, making the bump at $\Phi \sim 0.16$ closer to the inner turning point. The shortest possible trapping cycle of 2 modes is achieved for a feature in the middle at $\Phi \sim 0.5$, so moving a bump closer to an edge has the effect of making the trapping cycle longer. Finally, the lack of mode trapping in the $40 \%$ crystallized case is caused by the removal of the $\Phi \sim 0.16$ bump due to crystallization, phase separation, and the subsequent rehomogenization: the remaining bumps cause virtually no mode trapping.

As a final note we point out that the core/envelope symmetry found by Montgomery et al. (2003) is not an issue for these models, at least for modes with periods greater than $300 \mathrm{~s}$. This is because, as is conclusively demonstrated in Fig. 17, the outer chemical transition zones $(\mathrm{C} / \mathrm{He}$ and $\mathrm{He} / \mathrm{H})$ are found to be so smooth that they produce almost no mode trapping; thus, any mode trapping must be a result of structure in the deeper regions.

\section{Discussion and conclusions}

Convective overshooting is a longstanding problem in the theory of stellar structure and evolution. It is well known on theoretical grounds that during many stages in their lives stars experience overshoot episodes, that is, partial mixing beyond the formally convective boundaries as predicted by the 


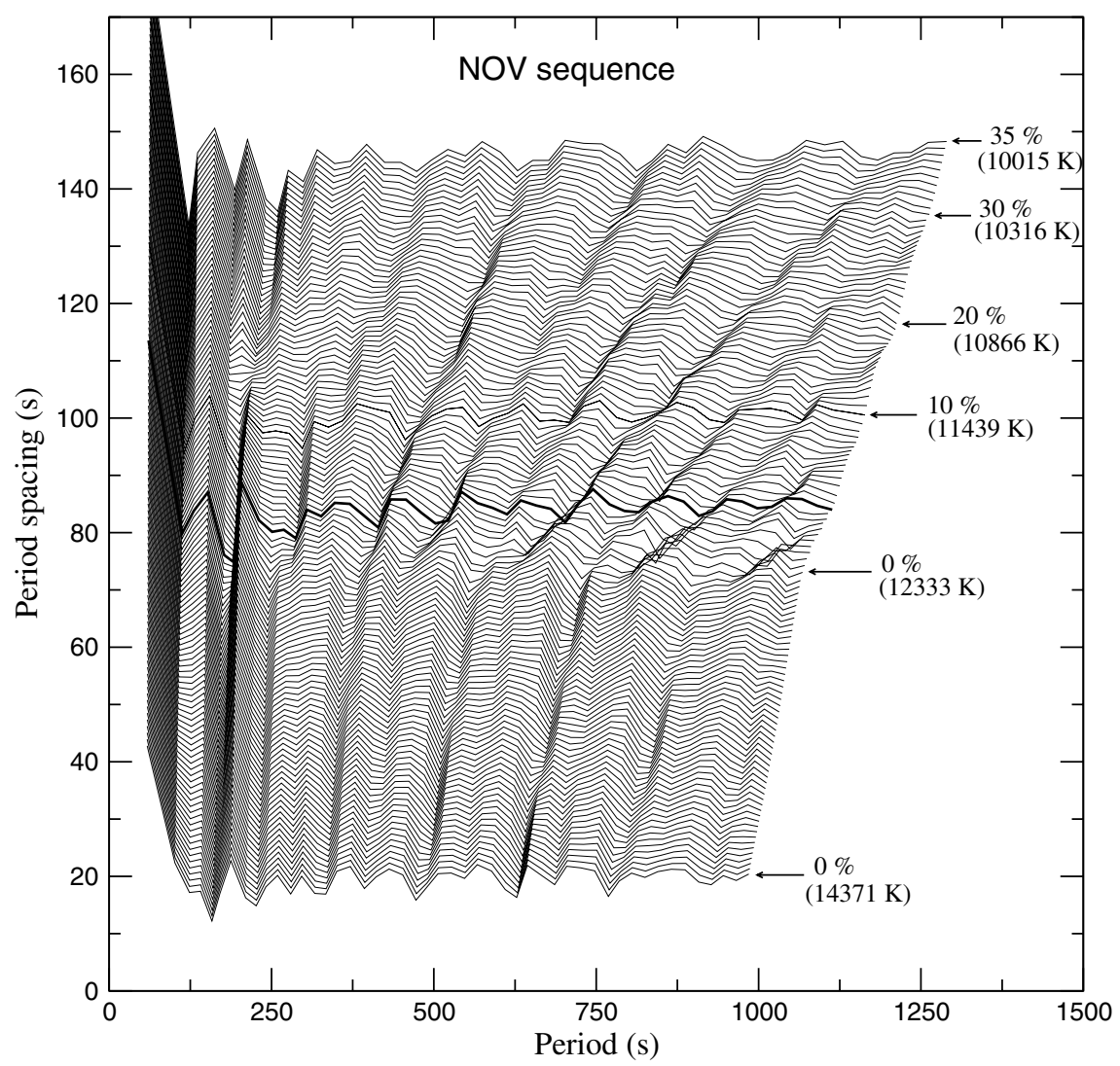

Fig. 14. Period spacing vs. period for effective temperature values between $\approx 15000$ and $10000 \mathrm{~K}$ corresponding to the NOV model sequence. In the interests of clarity, each curve has been shifted upward starting from the lowest one, in increments of $1 \mathrm{~s}$. For details, see the text.

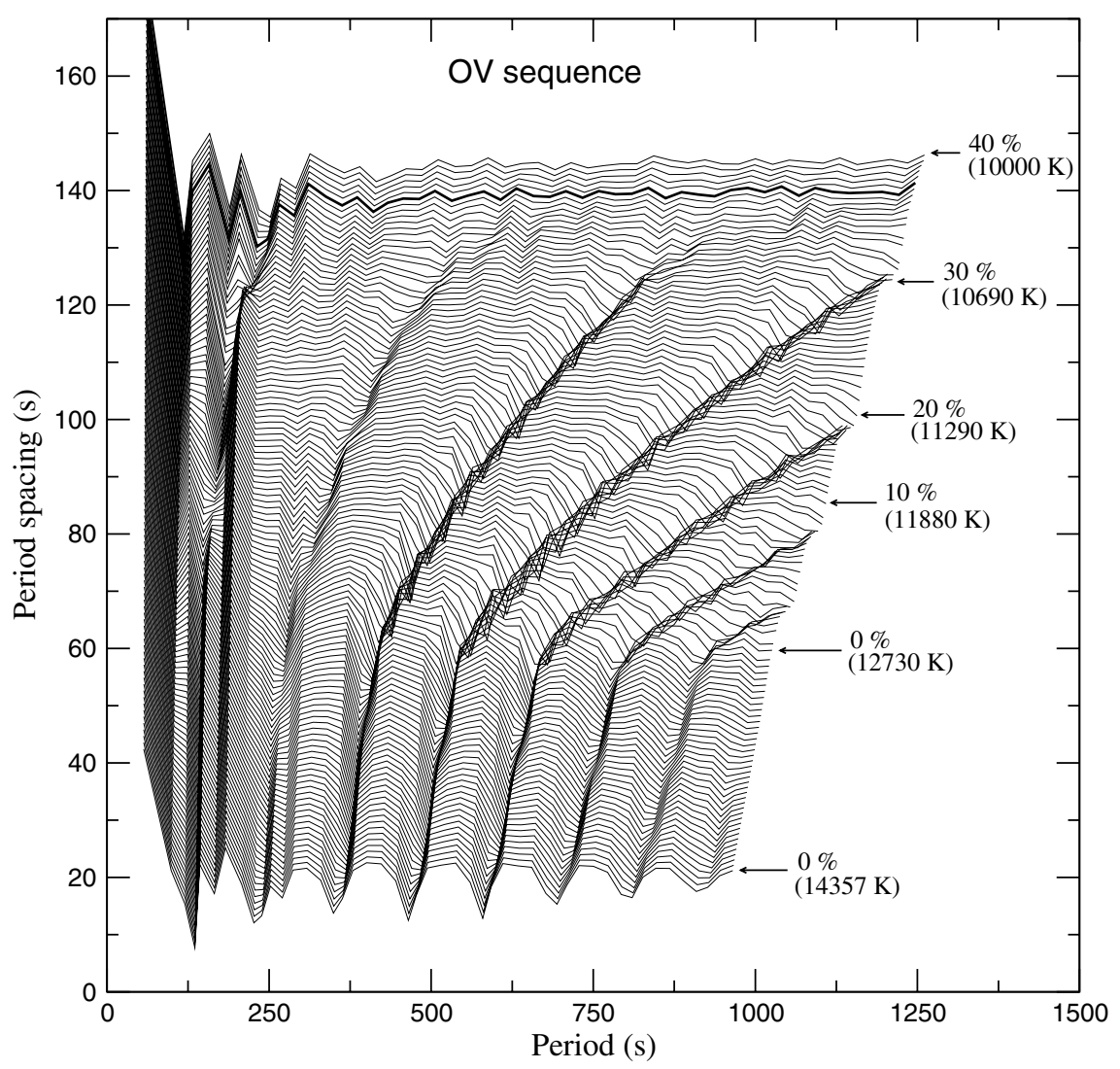

Fig. 15. Same as Fig. 14, but for the OV model sequence. 


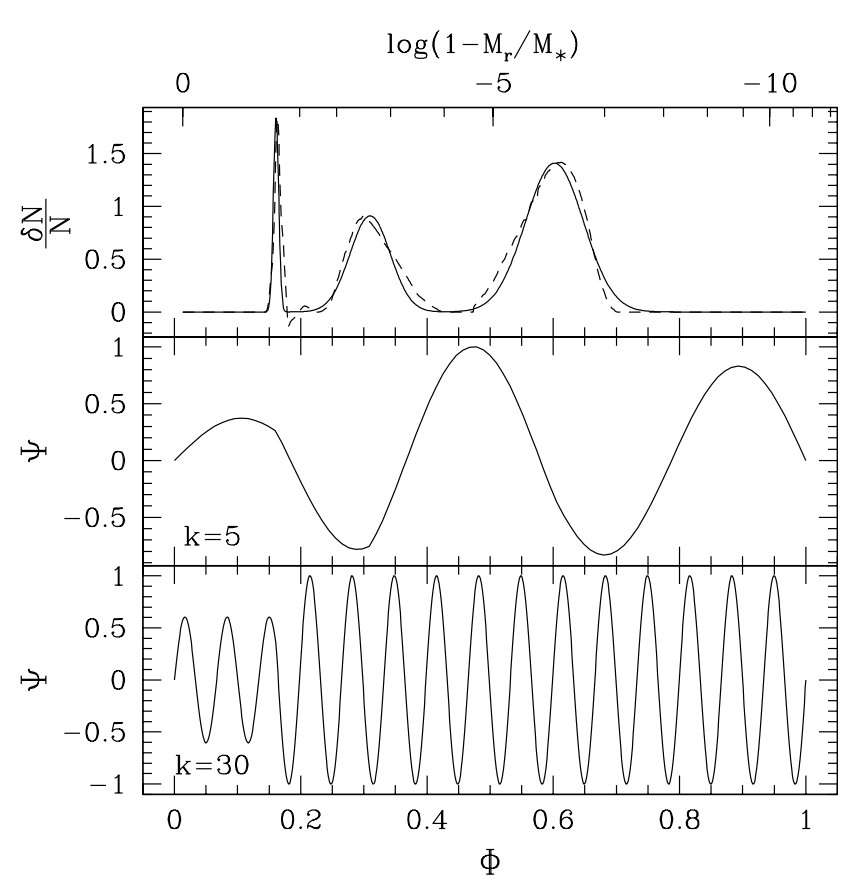

Fig. 16. Top panel: the bumps/non-smooth features in the BruntVäisälä frequency found in our numerical models (dashed curve) and the assumed bumps which we use in our simple analytical model (solid curve). In the lower panels, we illustrate how these features can act to trap modes. For instance, the narrowness of the $\Phi=0.16$ feature, compared to the spatial wavelength of these modes, causes it to induce amplitude changes in the two eigenmodes pictured.

Schwarzschild criterion of convective stability (Zahn 1991; Canuto 1992; Freytag et al. 1996; see also Renzini 1987). In particular, core overshooting taking place during central burning is an important issue because it has significant effects on the stellar structure and evolution. Over the years, considerable observational effort has been devoted to demonstrating the occurrence of core overshooting. Indeed, confrontation of stellar models with a wide variety of observational data suggests that convective overshoot takes place in real stars - see Stothers \& Chin (1992), Alongi et al. (1993), Kozhurina-Platais et al. (1997), Herwig et al. (1997), von Hippel \& Gilmore (2000), among others.

However, most of the evidence about the occurrence of core overshooting relies primarily on observational data from the very outer layers of stars from where radiation emerges. A more promising and direct way of placing constraints on the physical processes occurring in the very deep interior of stars is by means of the study of their pulsational properties. Pulsating white dwarfs are particularly important in this regard. In fact, white dwarfs constitute the end product of stellar evolution for the vast majority of stars, and the study of their oscillation spectrum through asteroseismological techniques has become a powerful tool for probing the otherwise inaccessible inner regions of these stars (Bradley 1998b; Metcalfe et al. 2002; Metcalfe 2003). White dwarf asteroseismology has also opened the door to peering into the physical processes that lead to the formation of these stars.

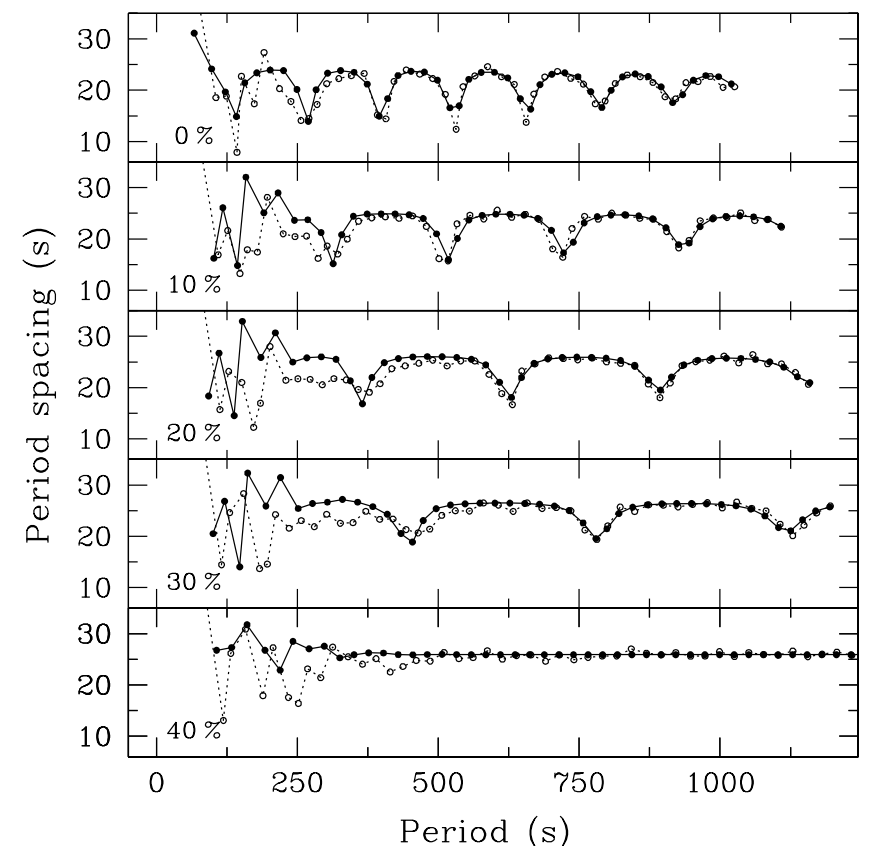

Fig. 17. Comparison of the mode trapping of the analytical model (filled circles, solid lines) to that of the full numerical results shown in Fig. 13 (open circles, dotted lines). Each panel is labelled with the crystallized mass fraction to which it corresponds.

In this work - and also in Paper I - we have argued that the occurrence of core overshoot episodes during core helium burning leaves strong imprints on the theoretical period spectrum of massive ZZ Ceti stars, features which could in principle be used for providing strong constraints on the occurrence of such episodes. On the other hand, we have demonstrated that the chemical rehomogenization induced by phase separation gives rise to a featureless period spectrum when the crystallized mass fraction is larger than $\approx 30 \%$. One of the aims of the present paper was to place constraints on the stellar mass and $T_{\text {eff }}$ values at which we should expect a pulsational pattern without any signature of core overshoot. We have seen that in our OV sequence the chemical discontinuity left by core overshooting is wiped out by chemical rehomogenization for a solid core larger than $37 \%$, which for a $0.94-M_{\odot}$ white dwarf takes place at $T_{\text {eff }}=10400 \mathrm{~K}$. The effective temperature at which this occurs is strongly dependent on the stellar mass. For instance, for 1.00-, 1.03-, and 1.05- $M_{\odot}$ white dwarf models, the traces of core overshooting are removed by chemical rehomogenization at $T_{\text {eff }}=11700,12350$, and $12800 \mathrm{~K}$, respectively.

The white dwarf star BPM 37093, the most massive ZZ Ceti known to date, is particularly noteworthy in this regard. Using IUE spectra, Koester \& Allard (2000) derive $T_{\text {eff }}=$ $11520 \mathrm{~K}, \log g=8.67$ and $M_{*}=1.03 M_{\odot}$. Other recent determinations place the mass of BPM 37093 at $1.00 M_{\odot}$ (Bergeron et al. 2001) and $1.10 M_{\odot}$ (Bergeron et al. 2004; Fontaine et al. 2003). Even given these uncertainties, it seems likely that its mass lies in the range $1.0-1.1 M_{\odot}$. With regard to its pulsation properties, BPM 37093 exhibits $g$-mode pulsations with periods in the range 500-660 s (Kanaan et al. 2000). The periods are very accurately determined, but as far as we are aware, 
the $\ell$ values are not securely identified ${ }^{6}$. On general grounds it is possible to show that the observed modes cannot all be $\ell=1$, so the simplest assumption has been that they are $\ell=2$ modes $^{7}$ (Nitta et al. 2000; Kanaan et al. 2000; Montgomery \& Winget 1999). Under this assumption it is possible to construct an "observed" period spacing diagram, which is characterized by an average period spacing of $17.125 \mathrm{~s}$ with deviations due to mode trapping of $\approx \pm 8 \mathrm{~s}$.

Given the uncertainties in the mass and $T_{\text {eff }}$ of BPM 37093 and also in its mode identification, we cannot make a definite statement concerning either whether core overshooting took place in BPM 37093 or what degree of crystallization it possesses. However, we can place constraints on the different possibilities. In fact, we can characterize BPM 37093 by a stellar mass in the range $1.00 M_{\odot} \lesssim M_{*} \lesssim 1.10 M_{\odot}$ and an effective temperature in the interval $11000 \lessgtr T_{\text {eff }} \lessgtr 12000 \mathrm{~K}$, given the present uncertainties in the derivation of these quantities. In the case of $1.00 M_{\odot}$ our treatment of crystallization predicts that the model should be $37 \%$ crystallized at $\approx 11700 \mathrm{~K}$. Thus, its observed frequency spectrum could reflect mode trapping effects due to prior evolutionary episodes of core overshoot. On the other hand, if BPM 37093 has a mass of $M_{*} \approx 1.10 M_{\odot}$, then there should be virtually no mode trapping features in its period spectrum. In this case we cannot say anything about the occurrence of core overshoot during pre-white dwarf evolution. A possible explanation of the observed period spectrum would be that the observed modes are not all $\ell=2, m=0$ modes, but instead a mixture of $\ell=1$ and $\ell=2$ modes (Metcalfe et al. 2004; Nitta et al. 2000).

\section{Concluding remarks}

This paper is aimed at specifically assessing the feasibility of employing white dwarf asteroseismology to demonstrate the occurrence of core overshooting during core helium burning. The investigation is focused on, intermediate-mass stars, the complete evolution of which has been followed from the zeroage main sequence through the thermally pulsing phase on the asymptotic giant branch (AGB) to the white dwarf regime. This allows us to obtain ZZ Ceti models with a physically sound internal structure consistent with the predictions of stellar evolution theory. Our work shows that for studying the effects of core overshooting, pulsating white dwarfs with masses of $\sim 0.90 M_{\odot}$ would be ideal since the potential for crystallization will not be a factor in erasing the signature of these overshooting events. Conversely, for studying the effects of crystallization, masses $\gtrsim 1.0 M_{\odot}$ are best since most of the core structure will be erased by crystallization and the chemical mixing associated with phase separation, making it easier to isolate and study the effect of crystallization on its own. In future studies we will focus on models in these mass ranges.

\footnotetext{
${ }^{6}$ By applying the $\ell$-value identification method of Robinson et al. (1995), Nitta et al. (2000) have discarded the possibility that the main periods observed in BPM 37093 are $\ell=3$ modes, but they cannot give definite identifications regarding $\ell=1$ or $\ell=2$ values.

7 If all the modes are $\ell=1$ then the average period spacing would imply a mass much larger than the values found from the spectroscopic determinations.
}

Acknowledgements. We warmly acknowledge to our referee Donald Winget for his suggestions and comments that improved the original version of this work. This research was supported by the Instituto de Astrofísica La Plata, by the UK Particle Physics and Astronomy Research Council, by the CIRIT, by MCYT grants AYA2002-04094-C03-01 and 02, and by the European Union FEDER funds. LGA also acknowledges the Spanish MCYT for a Ramón y Cajal fellowship.

\section{References}

Abrikosov, A. A. 1960, Sov. Phys. JETP, 12, 1254

Aizenman, M., Smeyers, P., \& Weigert, A. 1977, A\&A, 58, 41

Alexander, D. R., \& Ferguson, J. W. 1994, ApJ, 437, 879

Alongi, M., Bertelli, G., Bressan, A., et al. 1993, A\&A, 97, 851

Althaus, L. G., Serenelli, A. M., Córsico, A. H., \& Benvenuto, O. G. 2002, MNRAS, 330, 685

Althaus, L. G., Serenelli, A. M., Córsico, A. H., \& Montgomery, M. H. 2003, A\&A, 404, 593 (Paper I)

Angulo, C., Arnould, M., Rayet, M., et al. 1999, Nucl. Phys. A, 656, 3

Arnett, W. D., \& Truran, J. W. 1969, ApJ, 157, 339

Bergeron, P., Leggett, S. K., \& Ruiz, M. T. 2001, ApJS, 133, 413

Bergeron, P., Fontaine, G., Billères, M., Boudreault, S., \& Green, E. M. 2004, ApJ, 600, 404

Bradley, P. A. 1996, ApJ, 468, 350

Bradley, P. A. 1998a, ApJS, 116, 307

Bradley, P. A. 1998b, Baltic Astron., 7, 111

Bradley, P. A. 2001, ApJ, 552, 326

Brassard, P., Fontaine, G., Pelletier, C., \& Wesemael, F. 1991a, in White Dwarfs, ed. G. Vauclair, \& E. Sion (Kluwer Academic Publishers), 193

Brassard, P., Fontaine, G., Wesemael, F., Kawaler, S. D., \& Tassoul, M. 1991b, ApJ, 367, 601

Brassard, P., Fontaine, G., Wesemael, F., \& Hansen, C. J. 1992a, ApJS, 80, 369

Brassard, P., Fontaine, G., Wesemael, F., \& Tassoul, M. 1992b, ApJS, 81,747

Brickhill, A. J. 1991, MNRAS, 251, 673

Burgers, J. M. 1969, Flow Equations for Composite Gases (New York: Academic)

Canuto, V. M. 1992, ApJ, 392, 218

Caughlan, G. R., \& Fowler, W. A. 1988, Atomic Data and Nuclear Data Tables, 40, 290

Córsico, A. H., Althaus, L. G., Benvenuto, O. G., \& Serenelli, A. M. 2001, A\&A, 380, L17

Córsico, A. H., Althaus, L. G., Benvenuto, O. G., \& Serenelli, A. M. 2002, A\&A, 387, 531

Cox, J. P. 1980, Theory of Stellar Pulsations (Princeton: Princeton University Press)

Deubner, F., \& Gough, D. 1984, ARA\&A, 22, 593

Dolez, N., \& Vauclair, G. 1981, A\&A, 102, 375

Fontaine, G., Bergeron, P., Billères, M., \& Charpinet, S. 2003, ApJ, 591,1184

Freytag, B., Ludwig, H. G., \& Steffen, M. 1996, A\&A, 313, 497

García-Berro, E., Hernanz, M., Mochkovitch, R., \& Isern, J. 1988, A\&A, 193, 141

Gautschy, A., \& Saio, H. 1995, ARA\&A, 33, 75

Gautschy, A., \& Saio, H. 1996, ARA\&A, 34, 551

Gautschy, A., Ludwig, H., \& Freytag, B. 1996, A\&A, 311, 493

Goldreich, P., \& Wu, Y. 1999, ApJ, 511, 904

Gough, D. O. 1993, in Astrophysical fluid dynamics, ed. J. P. Zahn, \& J. Zinn-Justin (Amsterdam: Elsevier Science Publishers), 399 
Grossman, S. A., \& Taam, R. E. 1996, MNRAS, 283, 1165

Grossman, S. A., Narayan, R., \& Arnett, D. 1993, ApJ, 407, 284

Herwig, F., Blöcker, T., Schönberner, D., \& El Eid, M. 1997, A\&A, 324, L81

Iglesias, C. A., \& Rogers, F. J. 1996, ApJ, 464, 943

Isern, J., Mochkovitch, R., García-Berro, E., \& Hernanz, M. 1997, ApJ, 485, 308

Ichimaru, S., Iyetomi, H., \& Ogata, S. 1988, ApJ, 334, L17

Kanaan, A., Kepler, S. O., Giovannini, O., \& Diaz, M. 1992, ApJ, 390, L89

Kanaan, A., Nitta, A., Winget, D. E., et al. 2000, Baltic Astron., 9, 87

Kawaler, S. D., Winget, D. E., \& Hansen, C. J. 1985, ApJ, 295, 547

Kirzhnits, D. A. 1960, Sov. Phys. JETP, 11, 365

Koester, D., \& Allard, N. F. 2000, Baltic Astron., 9, 119

Kotak, R., van Kerkwijk, M. H., \& Clemens, J. C. 2002, A\&A, 388, 219

Kozhurina-Platais, V., Demarque, P., Platais, I., Orosz, J. A., \& Barnes, S. 1997, 113, 1045

Magni, G., \& Mazzitelli, I. 1979, A\&A, 72, 134

Metcalfe, T. S., Montgomery, M. H., \& Kanaan, A. 2004, ApJ, L133

Metcalfe, T. S. 2003, ApJ, 587, L43

Metcalfe, T. S., Salaris, M., \& Winget, D. E. 2002, ApJ, 573, 803

Montgomery, M. H., Klumpe, E. W., Winget, D. E., \& Wood, M. A. 1999, ApJ, 525, 482

Montgomery, M. H., Metcalfe, T. S., \& Winget, D. E. 2003, MNRAS, 344,657

Montgomery, M. H., \& Winget, D. E. 1999, ApJ, 526, 976

Nitta, A., Kanaan, A., Kepler, S. O., et al. 2000, Baltic Astron., 9, 97

Ogata, S., \& Ichimaru, S. 1987, Phys. Rev. A, 36, 5451

Osaki, Y., \& Hansen, C. J. 1973, ApJ, 185, 277

Pfeiffer, B., Vauclair, G., Dolez, N., et al. 1996, A\&A, 314, 182
Renzini, A. 1987, A\&A, 188, 49

Robinson, E. L., Mailloux, T., Zhang, E., et al. 1995, ApJ, 438, 908

Salaris, M., Domínguez, I., García-Berro, E., et al. 1997, ApJ, 486, 413

Salaris, M., García-Berro, E., Hernanz, M., Isern, J., \& Saumon, D. 2000, ApJ, 544, 1036

Salpeter, E. E. 1961, ApJ, 134, 669

Segretain, L., \& Chabrier, G. 1993, A\&A, 271, L13

Stevenson, D. J. 1980, J. Phys. Suppl., 41, C2-C53

Stothers, R. B., \& Chin, C. W. 1992, ApJ, 390, 136

Straniero, O., Domínguez, I., Imbriani, G., \& Piersanti, L. 2003, ApJ, 583,878

Sringfellow, G. S., De Witt, H. E., \& Slattery, W. L. 1990, Phys. Rev. A, 41, 1105

Tassoul, M., Fontaine, G., \& Winget, D. E. 1990, ApJS, 72, 335

Unno, W., Osaki, Y., Ando, H., Saio, H., \& Shibahashi, H. 1989, Nonradial Oscillations of Stars (University of Tokyo Press), 2nd edition

Van Horn, H. M. 1968, ApJ, 151, 227

van Kerkwijk, M. H., Clemens, J. C., \& Wu, Y. 2000, MNRAS, 314, 209

von Hippel, T., \& Gilmore, G. 2000, AJ, 120, 1384

Winget, D. E., Kepler, S. O., Kanaan, A., Montgomery, M. H., \& Giovannini, O. 1997, ApJ, 487, L191

Winget, D. E., Van Horn, H. M., Tassoul, M., et al. 1982, ApJ, 252, L65

Wood, M. A., \& Winget, D. E. 1988, In Proc. Multimode Stellar Pulsations Workshop, ed. G. Kovács, L. Szabados, \& B. Szeidl (Budapest: Konkoly Obs.), 199

Zahn, J.-P. 1991, A\&A, 252, 179 